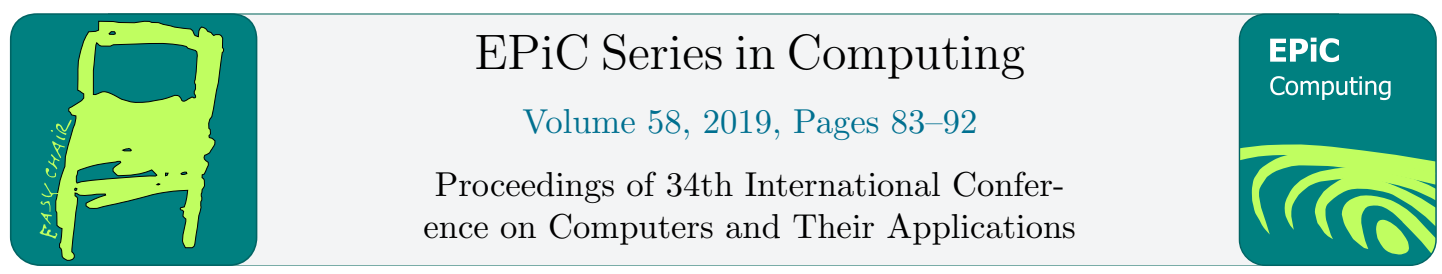

\title{
A Novel Multi-radio Rendezvous Algorithm for Cognitive Radio Networks
}

\author{
Michael Fischer ${ }^{1}$ and Jonathan Backens ${ }^{1}$ \\ Christopher Newport University, Newport News, Virginia, USA \\ (michael.fischer.14, jonathan.backens)@cnu.edu
}

\begin{abstract}
Channel Rendezvous between secondary users remains a key challenge to the development of cognitive ad-hoc networks. The decentralized and heterogeneous nature of ad-hoc CRNs makes guaranteeing rendezvous across multiple users within a short time difficult. Current research focuses on single hop networks or on multi-radio platforms to reduce the Time To Rendezvous (TTR). This work presents a Novel Multi-radio Rendezvous algorithm that leverages increasing availability of multi-radio secondary users to reduce TTR in heterogeneous and anonymous CRNs with multiple users.
\end{abstract}

\section{Introduction}

Cognitive Radio Networks (CRN) provide a platform for addressing the under utilization of the licensed spectra through a variety of Dynamic Spectrum Access (DSA) techniques[9][10]. In such environments, Secondary Users (SUs) are allowed to access licensed Primary Users (PU) spectra provided that they do not interfere with PU network operations. Since the SUs access to the licensed spectra is opportunistic, obtaining a shared communication channel between SUs must occur before any data transmissions can be undertaken. Therefore channel rendezvous plays a crucial role in network connectivity[5].

A traditional approach to solving the channel rendezvous problem involves the use of a reserved common control channel (CCC) available to all SUs for coordination. However the availability of CCC in licensed spectra may be compromised since it will be subject to dynamic PU activity. If the CCC is assumed instead to be in unlicensed spectra then it may be subject to congestion or interruption from other unlicensed users not in the CRN and potential jamming by malicious users.

Solutions to the rendezvous problem can also be found using centralized algorithms by assuming a priori knowledge of the available channel sets at each user or a priori knowledge of predetermined asymmetric roles each SU may be assigned. Although both of these assumptions can reduce the rendezvous latency, such knowledge is unrealistic in practice. Therefore decentralized algorithms with symmetric-role SUs are more practical and will be explored in this work.

In the scenario were symmetric channel sets are available to each $\mathrm{SU}$, the process of finding a rendezvous channel for communications becomes simpler. However, in practice SUs may 
have significant differences in both hardware capabilities and local spectrum conditions. Thus asymmetric channel sets are more commonly considered. Since the SUs have no previously established coordination, there is no consensus about which channels to switch onto in order to establish network communications during initial network establishment or after an interrupting PU event.

This paper will address some of these challenges by presenting a Multi-radio Rendezvous Algorithm that leverages the increased availability of multi-radio SUs to reduce Time to Rendezvous in CRNs with multiple users. Section 2 will presented the related research on approaches to solve the rendezvous problem. Section 3 will present the MPRS algorithm and give a short example of the rationale. Section 4 presents the theoretical performance of the MPRS algorithm in terms of Maximum and Expected Time to Rendezvous. Section 5 presents Simulation results of the algorithms performance. Finally, Section 6 concludes this work.

\section{Related Work}

Algorithms that rendezvous several users, are commonly known as a multi-rendezvous algorithms. A multi-rendezvous algorithms for the emphblind rendezvous problem (no CCC or centralized controller) has not been extensively studied by researchers. Current algorithms suitable for multi-rendezvous utilize concepts of traditional, user-to-user rendezvous and either add or adapt features to scale the solution[7, 15, 2]. Most rendezvous algorithms rely on a channel hopping scheme where users "hop" onto different frequencies in a specified sequence or pattern in order to achieve rendezvous[13, 4, 12]. Some classic blind rendezvous algorithms that use channel hopping sequences are the Generated Orthogonal Sequence, Modular Clock, and Modified Modular Clock algorithms, etc. [11]. These hop sequences are a critical aspect to the success of the rendezvous algorithm, and can be extended to the multi-user scenario.

Currently, one of the more widely used algorithms is the Jump-Stay algorithm[7], which extends the Modular Clock algorithm to provide guaranteed rendezvous; something that the Modular Clock algorithm could not guarantee. The Jump-Stay algorithm was designed to be used in a CRN that does not require time synchronization between users, intended to be applicable in both symmetric and asymmetric model conditions, and is suitable for multi-user rendezvous.

Gandhi et al. [2] presents an optimization to the Jump-Stay algorithm by exploiting redundancy and symmetry. Another widely used algorithm is the novel Ring Walk algorithm, [6] that provides a unique graph theory solution based on traversing a ring at different speeds. Each user has a different velocity of travel that is a key component to guaranteeing rendezvous.

The role-based model is a scheme where users assume different "roles" to play in the rendezvous process, such as the user who searches for the other and the user whose role designates he stays on one channel and waits. A Simple Role-Based model is presented in [3], which relies on the characteristics of the symmetric model to achieve rendezvous. Another symmetric rendezvous model, the Adaptive Multiple Rendezvous Control Channel algorithm, operates by providing a dynamic control channel and a protocol for nodes to enter and leave the network [1]. While these examples are not considered solutions to the blind rendezvous problem, they do illustrate the successful extension of other rendezvous algorithms and concepts to achieve rendezvous for multiple users in a CRN.

New research involving cognitive users equipped with multiple radios presents new opportunities to adapt algorithms to perform multiple rendezvous when they were previously incapable, or even perform rendezvous in parallel utilizing the additional hardware [15, 8, 14]. 


\section{Multi-Radio Parallel Rendezvous Algorithm}

To solve the multi-user rendezvous problem, we present a novel adaptation of the decentralized RPS algorithm termed Multi-Radio Parallel Rendezvous Scheme (MPRS) that includes multiradio equipped heterogeneous SUs. Presented in Algorithm 1, the MPRS algorithm provides guaranteed global rendezvous for all users in finite time, provided that they have at least one common channel.

\subsection{Algorithm Design}

The MPRS algorithm uses a channel hopping method of rendezvous, coupled with a role-based approach for the multiple radio devices of each user. A distinct channel sequence generation algorithm is used to produce the hopping sequences for each user in a round-robin manner, while also assigning channels to the radios of each user according to the role of each radio. Communication between users after they have achieved rendezvous on a commonly available channel, allows for the users to perform cooperative rendezvous until all users have achieved rendezvous and are using the same communications channel globally.

Like the RPS algorithm in [14] the MPRS algorithm also uses a role-based approach to assigning radios. Each user assigns one dedicated radio $S_{t m}^{i}$ and the remaining available radios as general radios $S_{t z}^{i}$.

Under the MPRS algorithm, once two users have established rendezvous on a common channel in their respective available channel sets, the two users then form a secondary set of channels that they both have in common, as can be seen in line 29 of Algorithm 1. The two users then utilize this secondary set of channels to reassign their dedicated radios, forming a common channel hopping sequence using shared $t_{\max }, m_{\text {total }}$ for the dedicated radio to step through (lines 16-18). This common channel set ensures that the two users will not lose communication since the dedicated radios will now have the same hopping sequence.

The dedicated radio assignment of all rendezvoused users occurs beginning at line 15 in Algorithm 1. Two separate assignment strategies exist in the MPRS algorithm dependent on whether or not the user has already achieved rendezvous with one or more users. For those users who have already established a previous connection, their dedicated radio channel is determined using the equation in line 16 of Algorithm 1. This modified equation uses the largest time slot value of all users who have already achieved rendezvous, $t_{\max }$, in addition to the sum of all radios of all such users as well, $m_{\text {total }}$.

Once channel sets have been exchanged, users have logically combined their general radios to form a virtual aggregated user and thus reduced the search rounds and decreasing TTR. By using this approach, the MPRS algorithm will continue to add users to the rendezvous set mathbf $N_{z}$ after each rendezvous to the accumulated set of users until all users have reached a common channel and achieved global rendezvous on a common set of channels. The MPRS algorithm covers both the symmetric and asymmetric cases of rendezvous. The algorithm handles different sets of channels by checking the common sets of channels for each user each time the MPRS algorithm attempts rendezvous. This way, if a user with a different set of common channels rendezvous with another user, the intersection of their two sets is taken to be used as the set of commonly available dedicated radio channels.

\subsection{A simple example}

To illustrate the operation of the MPRS algorithm, an example is presented in Figure 1. The example is comprised of a set of $|\mathbf{C}|=10$ channels and $K=3$ users each with $m=3$ radios. 


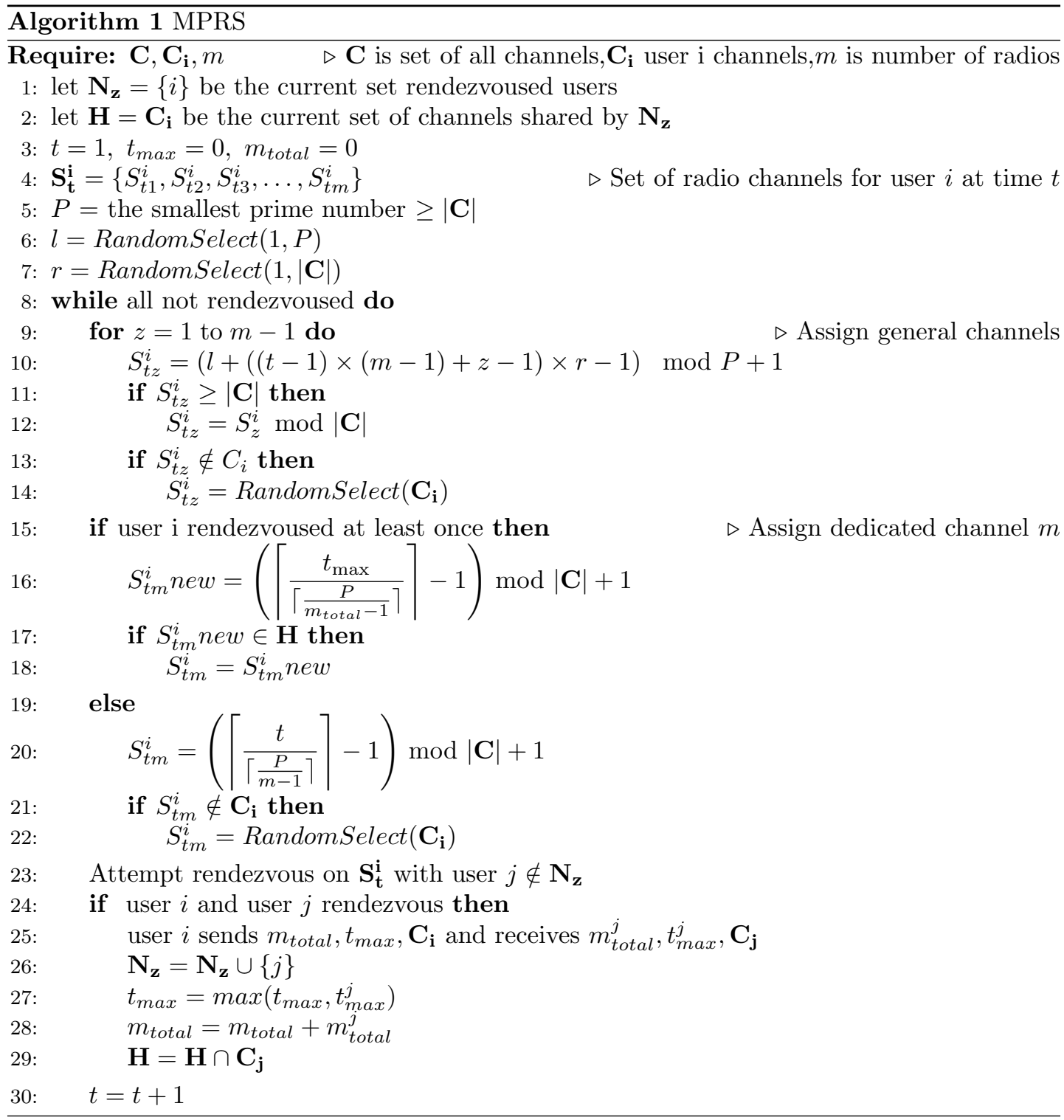

The symmetric model will be used for the example; meaning that all users will have access to all channels $\left(\left|\mathbf{C}_{\mathbf{i}}\right|=10\right.$. Assume all users are starting with their dedicated radio on a random channel in their available channel set $C_{i}$. For the purposes of the illustration, also assume user $1_{t}>$ user $2_{t}>$ user $3_{t}$, meaning the current time slot for User 1 is greater than that of User 2 and User 3.

Let the length of each round be $\left\lceil\frac{P}{m-1}\right\rceil=5$ time slots. The first rendezvous occurs at event $A$ between the general radios of User 1 and User 2 . User 1 and User 2 will then compare their sets of available channels and form a common set to use for their dedicated radios. The two users will also compare their time slot values and agree to use the largest of the two values as the shared time slot value. The length of the round for User 1 and User 2 has also decreased 


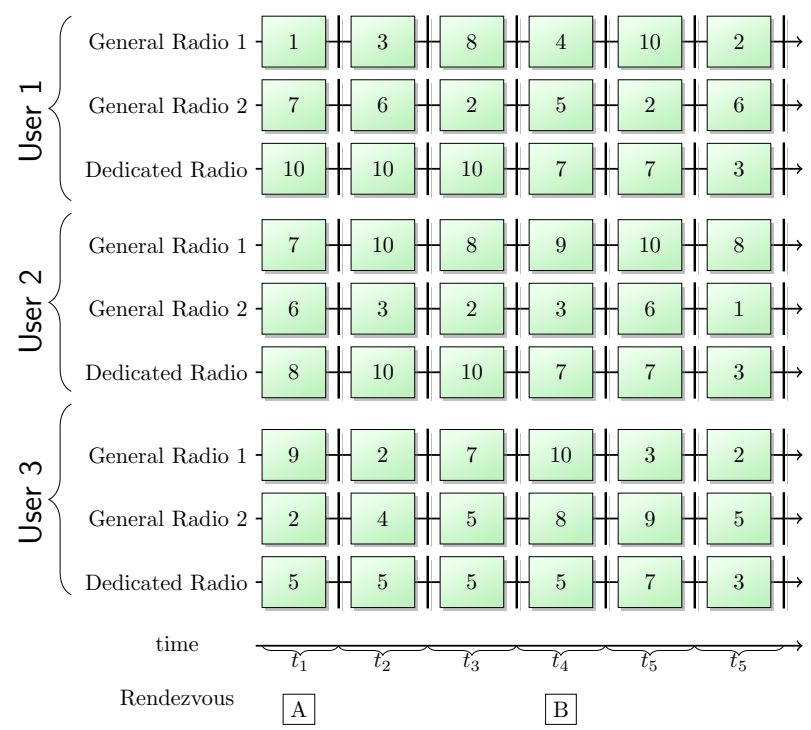

Figure 1: A working example of multi-user rendezvous using the MPRS algorithm

by time slot $t_{2}$ to $\left\lceil\frac{P}{m-1}\right\rceil=3$ because the number of radios between the 2 users has increased to a total of 6 .

The next rendezvous occurs between the dedicated radio of User 3 and general radio 2 of User 1 at event $B$ in time slot $t_{4}$. Note that, although User 3 did not finish its round of $\left\lceil\frac{P}{m-1}\right\rceil=5$ time slots, it joined the aggregate list of users who have already rendezvoused and added its radios to the total count, which influences the length of the round. Now the total length of each round for the dedicated radio of each user is $\left\lceil\frac{P}{9-1}\right\rceil=2$ at time slot $t_{4}$ with the addition of User 3.

\section{Theoretical Analysis}

The performance of the MPRS algorithm is evaluated using Maximum Time to Rendezvous (MTTR), the worst case TTR, and Expected Time to Rendezvous (ETTR), the average worst case of TTR. Both of these are determined for the two user case and then extended to K users.

Consider two users scenario, with users A and B in a heterogeneous network. Let user A have $m$ radios and user $\mathrm{B}$ have $n$ while assuming $m>n$ for the purposes of illustration. Since user round length is inversely proportional to the number of radios, user A will have a shorter round than that of user B and thus cycle through the available channels faster.
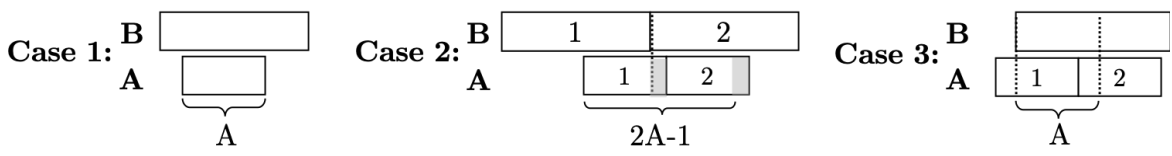

Figure 2: Two User Rendezvous Conditions

Figure 2 shows the possible rendezvous scenarios between user $\mathrm{A}$ and $\mathrm{B}$ when $m>n$. 
For the following analysis, let $A=\left\lceil\frac{P}{\max \{m, n\}-1}\right\rceil$ denote the round length of user A, and $B=\left\lceil\frac{P}{\min \{m, n\}-1}\right\rceil$ denote the round length of user $\mathrm{B}$, noting that $A<B$. The time to rendezvous is measured beginning from when both users appear on the network and have a possibility to reach a common channel. This leads to the following three cases.

Case 1. User A cycles through of all its available channels $\mathbf{C}_{\mathbf{A}}$ before user $\mathrm{B}$ changes its dedicated radio channel. Therefore, the worst case for rendezvous between both users would be the length of time it takes for user A to cycle through all of its channels, assuming in the worst case that the very last channel in $\mathbf{C}_{\mathbf{A}}$ is also the dedicated radio channel of $\mathrm{B}$. Therefore, for Case $1 M T T R=A$. In order to determine the probability in which the MTTR will be equal to $\mathrm{A}$, the occurrence probability of $\mathrm{A}$ can be determined $P(T T R=A)=\frac{B-A+1}{A+B-1}$ and thus $E T T R=\frac{B-A+1}{A+B-1}(A)$

Case 2. Case 2 illustrates the worst case scenario in which user A's round does not complete during user B initial round and thus user A overlaps with two rounds from user B. In the worst case user A finds user B's dedicated radio at the of end of $C_{A}$. However User B changes dedicated radio channels one time slot before user A reaches the channel. User A must then go through another complete round of its length minus one channel to reach the new dedicated radio channel of user B. Thus, the MTTR of Case 2 is $M T T R=2 A-1$ and the probability of this worst case occuring is $P(T T R=2 A-1)=\frac{A-1}{A+B-1}$. Thus for Case 2 ETTR $=\frac{A-1}{A+B-1}(2 A-1)$.

Case 3. User B appears after user A has begun searching through all of the channels in $C_{A}$. The worst case TTR in this scenario would be if the dedicated radio channel of user B occurs at the beginning of $C_{A}$, in which user $\mathrm{A}$ had already cycled through before user B appeared on the network. User A must now cycle through all of its channels again in order to reach the common channel at the beginning of its sequence to rendezvous with user B. Recalling that TTR is measured from when both users appear on the network, the TTR count would start from when user B appears and last for the number of time slots equivalent to the length of user A's round. Thus $M T T R=A$ and the probability $P(T T R=A)=\frac{A-1}{A+B-1}$. For Case 3 the ETTR would be $E T T R=\frac{A-1}{A+B-1}(A)$

Based on the above analysis, it can be seen that the maximum time it would take to achieve rendezvous is dominated by the user with the largest number of radios.

To generalize this MTTR analysis to any $K$ number of users, upper bounds for the MTTR for both the heterogeneous and homogeneous network environments can be found by observing the MPRS algorithms aggregation of $m_{t}$ otal after each rendezvous.

$$
\begin{gathered}
\text { heterogeneous: } \quad \text { MTTR }=\sum_{i=2}^{K} 2 \cdot\left\lceil\frac{P}{\max \left(m_{i}, \sum_{j=1}^{i-1} m_{j}\right)}\right\rceil-1 \\
\text { homogeneous : } \quad M T T R=\sum_{i=2}^{K-1}\left\lceil\frac{P}{i \cdot m}\right\rceil-1
\end{gathered}
$$

\section{Simulation}

Matlab was used to evaluate MPRS performance versus the popular JS algorithms. First the symmetric rendezvous model is evaluated in which all users have the same set of common 
channels.

The asymmetric rendezvous model describes a system in which users do not have the same number of channels commonly available. This introduces two new variables $\theta$ and $G$. The variable $\theta$ is defined as the percentage of channels available out of set $Q=|\mathbf{C}|$. The $G$ parameter defines the number of channels all users have commonly available in $\mathbf{C}$.

Multi-user JS vs MPRS (Symmetric)

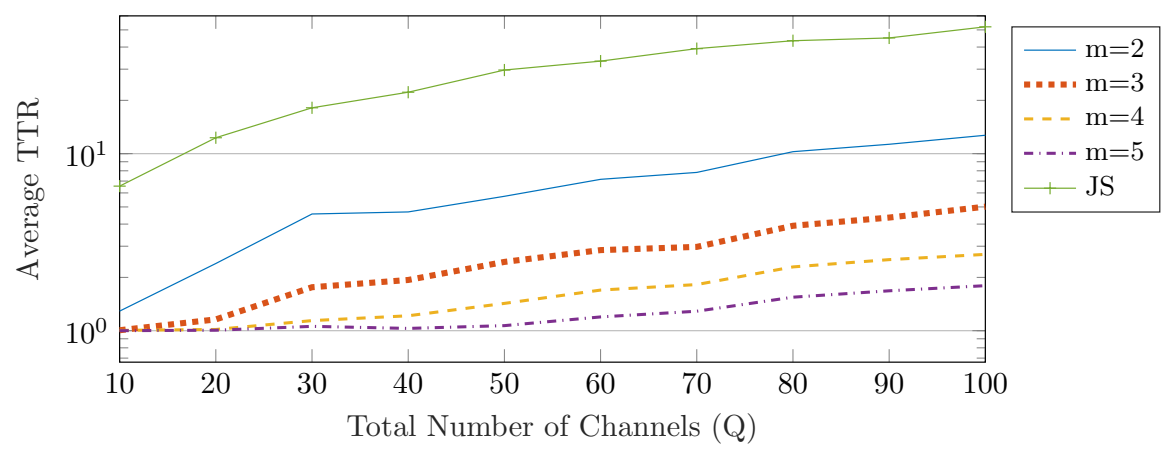

Multi-user JS vs MPRS (Symmetric)

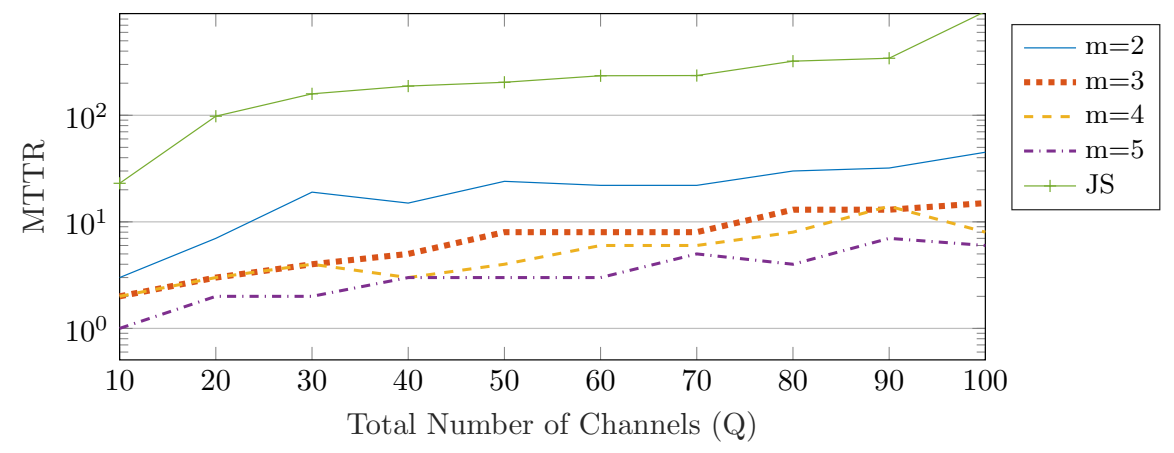

Figure 3: MPRS vs JS under a symmetric rendezvous model with $K=10$ users

Figure 3 illustrates the Average TTR and MTTR of the MPRS algorithm compared to that of the multi-user Jump-Stay algorithm under the symmetric rendezvous model. A clear difference in Average TTR and MTTR of the Jump-Stay algorithm can be seen, compared to that of the MPRS algorithm.

Figure 4 illustrates how the MPRS algorithm would scale with the addition of users. The total number of channels is kept constant at $Q=1000$ channels, and the number of users, $K$, is increased from 10 to 100 over time. A larger $Q$ value was chosen for this test to simulate a larger size network for scalability testing. As expected more users increases the total number of radios causing expected TTR and maximum TTR to decrease according to Equations 2.

Figure 5 illustrates different common channel allocation factors and the effects of such allocation on TTR with $\theta=0.2$. For this test, $Q=1000$ channels were used to ensure equal channel allocation for each user when taking the fractional portions of $G Q$ and $\theta Q$. As a result, this increase in total number of channels raises the average and maximum TTR values for the trial. A higher value of $G$ results in a decreased average and maximum TTR for all users due 


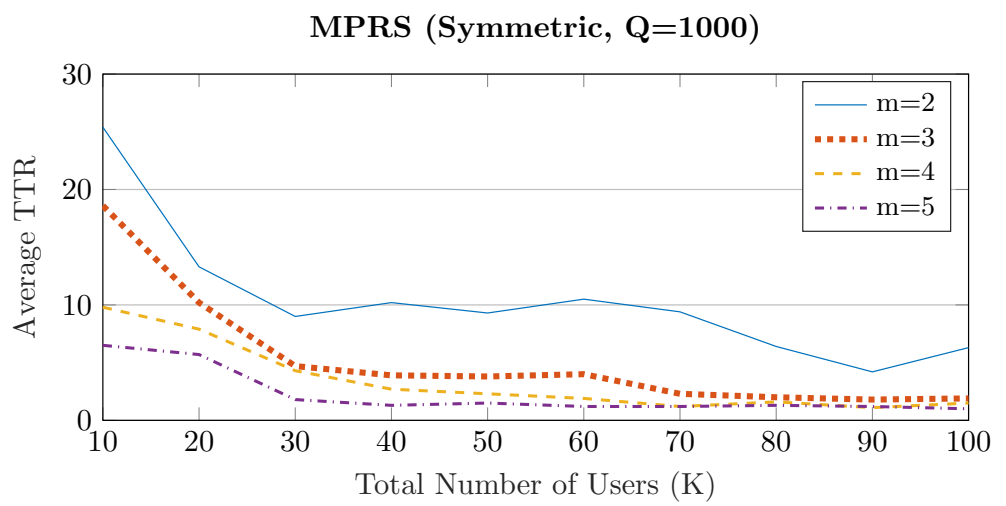

MPRS (Symmetric, Q=1000)

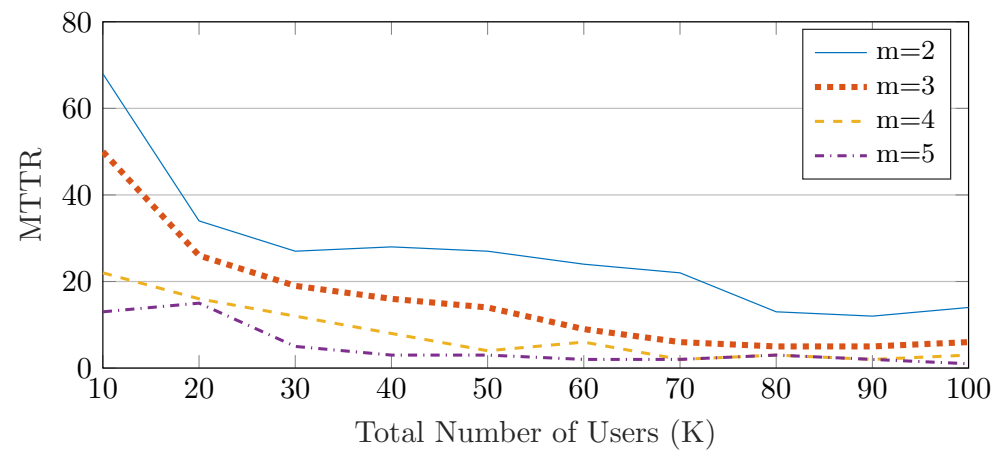

Figure 4: MPRS under a symmetric rendezvous model with increasing $K$

to the larger number of commonly available channels. As can be seen in Figure 5, a higher value of $G$ also corresponds to a slower increase in TTR as $Q$ increases. This effect causes the TTR values to initially begin with a smaller difference between each case, and end with a larger difference in TTR as $G$ increases.

\section{Conclusion}

In this paper, we have proposed a novel Multi-Radio Pairwise Rendezvous (MPRS) algorithm for homogeneous and heterogeneous CRNs under both symmetric and asymmetric channel conditions using a decentralized approach. Leveraging the increased flexibility of multiple radios for each user, the MPRS algorithm can greatly improve performance over traditional RPS rendezvous algorithms. Theoretical performance and extensive simulations have shown that MPRS has significant improvements in both MTTR and average TTR. In the future, we will continue to investigate exploiting multi-radio rendezvous under highly dynamic spectrum conditions in large multi-hop heterogeneous networks. 

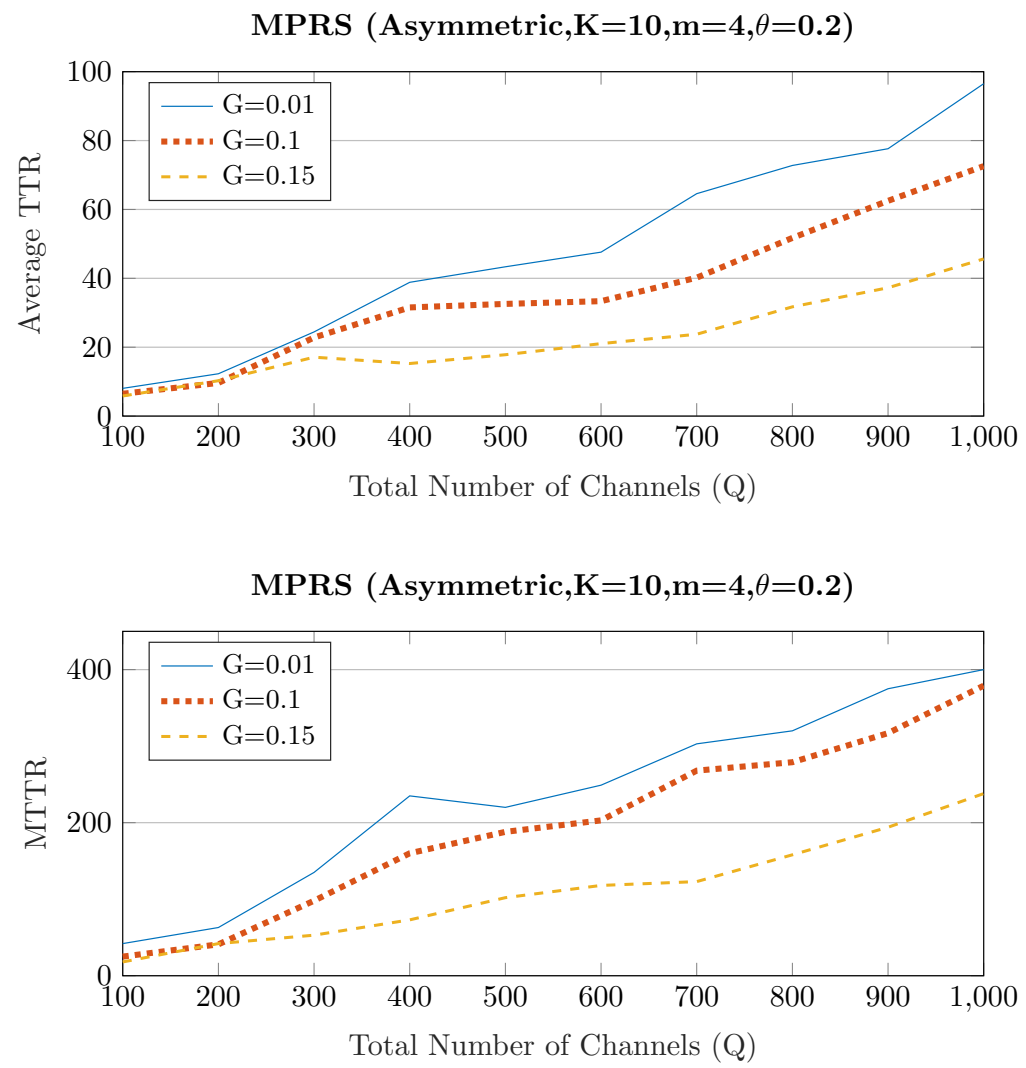

Figure 5: Average TTR with $m=4, K=10$, and varying common channel allocation

\section{References}

[1] Claudia Cormio and Kaushik. R. Chowdhury. An adaptive multiple rendezvous control channel for cognitive radio wireless ad hoc networks. In 2010 8th IEEE International Conference on Pervasive Computing and Communications Workshops, PERCOM Workshops 2010, pages 346-351. IEEE, mar 2010.

[2] Rohan Gandhi, Chih Chun Wang, and Y. Charlie Hu. Fast rendezvous for multiple clients for cognitive radios using coordinated channel hopping. In Annual IEEE Communications Society Conference on Sensor, Mesh and Ad Hoc Communications and Networks workshops, volume 1, pages 434-442. IEEE, jun 2012.

[3] E O Guerra, V A Reguera, R D Souza, G. Brante, and E M G Fernandez. Simple role-based rendezvous algorithm for cognitive ad hoc radio networks. Electronics Letters, 50(3):182-184, jan 2014.

[4] Kaigui Bian, Jung-Min Park, and Ruiliang Chen. Control Channel Establishment in Cognitive Radio Networks using Channel Hopping. IEEE Journal on Selected Areas in Communications, 29(4):689-703, apr 2011.

[5] Y. Liang, K. Chen, G. Y. Li, and P. Mahonen. Cognitive radio networking and communications: an overview. IEEE Transactions on Vehicular Technology, 60(7):3386-3407, Sept 2011.

[6] Hai Liu, Zhiyong Lin, Xiaowen Chu, and Yiu Wing Leung. Ring-walk based channel-hopping algorithms with guaranteed rendezvous for cognitive radio networks. In Proceedings - 2010 
IEEE/ACM International Conference on Green Computing and Communications, GreenCom 2010, 2010 IEEE/ACM International Conference on Cyber, Physical and Social Computing, CPSCom 2010, pages 755-760. IEEE, dec 2010.

[7] Hai Liu, Zhiyong Lin, Xiaowen Chu, Yiu-Wing Leung, Hai Liu, Xiaowen Chu, and Yiu-Wing Leung. Jump-Stay Rendezvous Algorithm for Cognitive Radio Networks. IEEE Transactions on Parallel and Distributed Systems, 23(10):1867-1881, oct 2012.

[8] Rajib Paul, Yalew Zelalem Jembre, and Young June Choi. Multi-interface rendezvous in selforganizing cognitive radio networks. In 2014 IEEE International Symposium on Dynamic Spectrum Access Networks, DYSPAN 2014, pages 531-540. IEEE, apr 2014.

[9] M. Song, C. Xin, Y. Zhao, and X. Cheng. Dynamic spectrum access: from cognitive radio to network radio. IEEE Wireless Communications, 19(1):23-29, February 2012.

[10] Carl R Stevenson, Gerald Chouinard, Zhongding Lei, Wendong Hu, Stephen J Shellhammer, and Winston Caldwell. Ieee 802.22: The first cognitive radio wireless regional area network standard. IEEE communications magazine, 47(1):130-138, 2009.

[11] Nick C Theis, Ryan W Thomas, and Luiz A DaSilva. Rendezvous for Cognitive Radios. IEEE Transactions on Mobile Computing, 10(2):216-227, feb 2011.

[12] Tsung Ying Wu, Wanjiun Liao, and Cheng Shang Chang. CACH: Cycle-Adjustable Channel hopping for control channel establishment in cognitive radio networks. In Proceedings - IEEE INFOCOM, 2014.

[13] Bo Yang, Wei Liang, Meng Zheng, and Ying Chang Liang. Fully Distributed Channel-Hopping Algorithms for Rendezvous Setup in Cognitive Multiradio Networks. IEEE Transactions on Vehicular Technology, 65(10):8629-8643, oct 2016.

[14] L. Yu, H. Liu, Y. Leung, X. Chu, and Z. Lin. Multiple radios for effective rendezvous in cognitive radio networks. In 2013 IEEE International Conference on Communications (ICC), pages 28572862, June 2013.

[15] Lu Yu, Hai Liu, Yiu Wing Leung, Xiaowen Chu, and Zhiyong Lin. Adjustable rendezvous in multiradio cognitive radio networks. In 2015 IEEE Global Communications Conference, GLOBECOM 2015, pages 1-7. IEEE, dec 2015. 